\title{
Lifelong exposures and the potential for stroke prevention: the contribution of cigarette smoking, exercise, and body fat
}

\author{
Roger Shinton
}

\begin{abstract}
Study objective - To examine the potential for stroke prevention by avoidance of hazards related to lifestyle. Assessment of the lifelong contribution of both individual and combined exposures was a particular feature of the study. An estimate was sought of the proportion (population attributable risk fraction) of strokes likely to be caused by one or a combination of cigarette smoking, lack of exercise, and obesity.

Design - Case-control study.

Setting - Eleven general practices in west Birmingham.
\end{abstract}

Participants - Altogether 125 men and women who had just had their first stroke and were aged 35-74 and 198 controls frequency matched for age and sex recruited over 24 months during 1988-90.

Main results - The hazards of cigarette smoking for stroke were confirmed and noted to persist for one to two decades after stopping smoking. Cigarette smoking was estimated to have caused $49 \%(95 \%$ confidence interval $22,67 \%$ ) of the strokes in this population with roughly equal contributions from current and former smoking. Combinations of cigarette smoking (current and former) with lack of exercise or previous obesity apparently caused $62 \%$ and $72 \%$ of the strokes respectively. If cigarette smoking, lack of exercise and obesity were all avoided, $79 \%(32,94 \%)$ of the strokes could potentially have been prevented. No stroke patient reported a combination of never smoking, taking regular exercise aged 15 to 25 , and avoidance of overweight (body mass index $>25 \mathrm{~kg} / \mathrm{m}^{2}$ ).

Conclusions - Taken together, the combination of cigarette smoking, excessive body fat, and lack of exercise accounted for a major proportion of stroke cases in the population studied. It appears that these easily identifiable factors related to lifestyle are a major and possibly predominant cause of stroke, at least until the age of 75 .

University of Birmingham, Department of Medicine (Elderly), Birmingham

Heartlands Hospital, Yardley Green Unit, Birmingham B9 5PX Roger Shinton

Correspondence to: Dr Shinton.
( $\mathcal{F}$ Epidemiol Community Health 1997;51:138-143)

The extent to which lifestyle can prevent stroke has received surprisingly little attention. Cigarette smoking is now widely recognised as a risk factor for stroke. ${ }^{1-4}$ There have, however, been few estimates of the proportion of strokes likely to have been caused by cigarette smoking in populations at risk (population attributable risk fraction). Considerable doubt also remains concerning the speed with which those stopping smoking return to the risk of those who never started. An Australian case-control study of hospital patients with "non-embolic" cerebral infarction has suggested the increased risk persists at least 10 years. $^{2}$ No increased risk of overall stroke, however, was seen in former smokers in the British regional heart study cohort and the risk quickly returned to baseline in the American nurses cohort. ${ }^{34}$

There is increasing evidence that other potentially avoidable hazards are also important causes of stroke, for example lack of exercise and excessive body fat. ${ }^{5-8}$ Case-control methods, allowing a lifelong view, have not yet reported the extent to which stroke is preventable by avoidance of particular combinations of hazards which are within the potential control of the individual. The west Birmingham stroke project was designed to examine the contribution of single and combined lifestyle related risk factors in causing stroke. Data from the study have already indicated that lifelong exercise and and the avoidance of overweight and obesity protect against the risk of stroke. ${ }^{78}$ The community setting of this study has the added advantage of allowing estimates of the proportion of strokes caused by particular exposures (the population attributable risk fraction) or combinations of exposures. These estimates, although strictly only appropriate for the study population, will reflect the picture in populations with similar characteristics.

\section{Methods}

The detailed methods of this case-control study have been previously described..$^{7-9}$ Those individuals currently on the register of 11 general practice partnerships in west Birmingham comprised the study base population $(n=101000)$.

\section{STROKE PATIENTS}

Stroke patients were assessed for inclusion using the standard World Health Organisation criteria - rapidly developing clinical signs of focal and at times global (applied to patients in deep coma and to those with subarachnoid haemorrhage) loss of cerebral function, with symptoms lasting more than 24 hours or leading to death, with no apparent cause other than that of vascular origin. ${ }^{10}$ Both hospital and 
community stroke patients, if fit, were offered a computerised tomography head scan (without contrast). Cerebral infarction was diagnosed if a head scan or autopsy was performed within 21 days of stroke onset and there was either no evidence of haemorrhage or a pattern of predominant infarction. Those included were male and female patients with their first stroke, between the ages of 35 and 74 years, occurring between 1st October 1988 and 30th September 1990. The following characteristics excluded stroke patients from the study; a prior history of stroke (but not transient ischaemic attack), a history of mitral valvular heart disease combined with atrial fibrillation, a history of primary or metastatic cerebral neoplasm, a history of coagulation disorder or myeloproliferative disease, and patients dying before assessment could be made.

CONTROL SUBJECTS

Subjects were randomly selected from the general practice population to match broadly by age and sex the incident stroke case distribution (frequency matching). The same exclusions applied as for stroke patients.

\section{ASSESSMENT}

The cases and controls were all anthropometrically assessed and interviewed by a single observer (RS) using a standard structured questionnaire. Stroke patients were seen as soon as possible following the event. When disability prevented an adequate response the closest relative or friend was interviewed.

Cigarette smoking status was classified into never, former, and current smokers by responses to to the questions; "Have you ever smoked cigarettes regularly, that is at least one cigarette per day for one year?" and "Have you smoked cigarettes during the past year?" Former smokers were also asked how long ago they stopped smoking. General practice and hospital notes were also examined for a prior record of cigarette smoking status.

To assess physical exercise, subjects were divided by their response to the question; "Did you often take part in vigorous sports or activities between the ages of 15 and 25 years?" Examples of vigorous exercise provided by the questionnaire were digging, running, swimming, cycling, playing tennis or squash, and keeping fit.

Standing height was measured, when possible, by standard methods using a portable stadiometer. ${ }^{11}$ Lying height was measured in all stroke patients admitted to hospital. For lying height the subject lay on his/her back and an assistant ensured the heels were correctly positioned at the base of the stadiometer. A simple regression equation which related standing and lying height amongst stroke patients who could stand enabled standing height to be estimated for those unable to stand. Cases and controls were asked; "What is the most you have ever weighed?" The response was converted into kilograms and combined with measured/derived standing height to provide maximum reported body mass index: weight in kilograms/(height in metres) ${ }^{2}$. Harpenden skinfold calipers were used to measure subscapular skinfold thickness using standard methods. ${ }^{11}$ Two records for each subject were made and the mean calculated. Sex specific interquartile ranges amongst controls were established to subdivide subjects by the various measures of body fat. Other variables which could confound or interact with observed relations were assessed by questionnaire and have been detailed previously. ${ }^{7-9}$

\section{STATISTICAL ANALYSIS}

A computer software programme $(E G R E T)^{12}$ using methods described by Breslow \& Day ${ }^{13}$ was employed to calculate odds ratios (relative risk) and their $95 \%$ confidence intervals. Mantel-Haentzel methods were used to provide an estimate of the odds ratio after adjustment for one or two other variables. Adjustment for several variables was made by use of the multiple logistic regression model. Regression models were fitted using exposure levels of a given risk factor as discrete (factored) variables. The population attributable risk fraction (PARF) and 95\% confidence intervals for a given exposure was estimated from the odds ratio and the prevalence of the exposure in the control population according to the equation ${ }^{14}$ :

$$
\text { PARF }=\frac{\text { prevalence }(\text { odds ratio }-1)}{\text { prevalence } \times(\text { odds ratio }-1)+1}
$$

Inspection of this formula demonstrates that raising either the odds ratio or the prevalence of the exposure will increase the size of the population attributable risk fraction. The population attributable risk fraction ranges from 0 (no effect) to 1 (all cases explained by the exposure)

\section{Results}

Altogether 125 eligible stroke patients were identified over 24 months. Forty three (34\%) were managed at home. No stroke patient (or relative) declined to participate. Cerebral infarction was diagnosed in 81 patients, intracerebral haemorrhage in five, and subarachnoid haemorrhage in four. In the remaining 35 patients computed tomography within 21 days was either impossible or declined by the patient, relative or attending physician. Twelve first event stroke patients were excluded, eight because they died before assessment was possible, three because of haematological disorders, and one due to mitral valve disease with atrial fibrillation. 198 (96\%) of the 207 eligible control subjects agreed to participate.

RECALL OF PREVIOUSLY RECORDED CIGARETTE SMOKING HISTORY

Cigarette smoking status had been recorded in medical records prior to the study assessment in 97 cases and 116 controls. Of the 75 cases 
Table 1 Relation between current and former cigarette smoking and stroke

\begin{tabular}{|c|c|c|c|c|c|c|}
\hline & \multicolumn{6}{|c|}{ Cigarette smoking status (OR $(95 \% C I))$} \\
\hline & Never & $E x>19 y$ & Ex $10-19 y$ & Ex $1-9 y$ & Current & Test for trend \\
\hline $\begin{array}{l}\text { Age and sex adjusted } \\
\text { Multiple risk factor adjusted* } \\
\text { Cases/controls }\end{array}$ & $\begin{array}{l}1.0 \\
1.0 \\
22 / 69\end{array}$ & $\begin{array}{l}1.85(0.61,5.80) \\
3.49(1.52,8.03) \\
15 / 20\end{array}$ & $\begin{array}{l}2.44(0.78,8.78) \\
2.29(0.86,6.10) \\
12 / 21\end{array}$ & $\begin{array}{l}3.43(1.37,8.76) \\
2.92(1.14,7.43) \\
27 / 28\end{array}$ & $\begin{array}{l}2.57(1.28,5.51) \\
3.02(1.48,6.13) \\
49 / 60\end{array}$ & $\mathrm{p}=0.002$ \\
\hline
\end{tabular}

* Covariables included in the logistic models were; age group, sex, race (black/non-black), vigorous exercise aged 15-25 and dietary saturated fat (four levels).

with a record of cigarette smoking, $72(96 \%)$ could recall a history at interview. Amongst the 81 controls with a recorded cigarette smoking history $79(98 \%)$ could recall the history at interview.

\section{CIGARETTE SMOKING AND STROKE}

Current smokers were grouped together for analysis but former smokers were subdivided into three groups by number of years since cigarette smoking cessation $(1-9,10-19$, and $>19$ ). This was to enable assessment of how soon after stopping smoking the risk of stroke declines to that of the never smoker. The age and sex adjusted odds ratios indicated the risk of stroke in current smokers was more than double that in never smokers (fig 1, table 1). The decrease in the risk with time since stopping smoking appears to be slow.

Multiple risk factor adjustment including vigorous exercise aged 15-25 and dietary saturated fat (4 levels) along with age group, sex, and

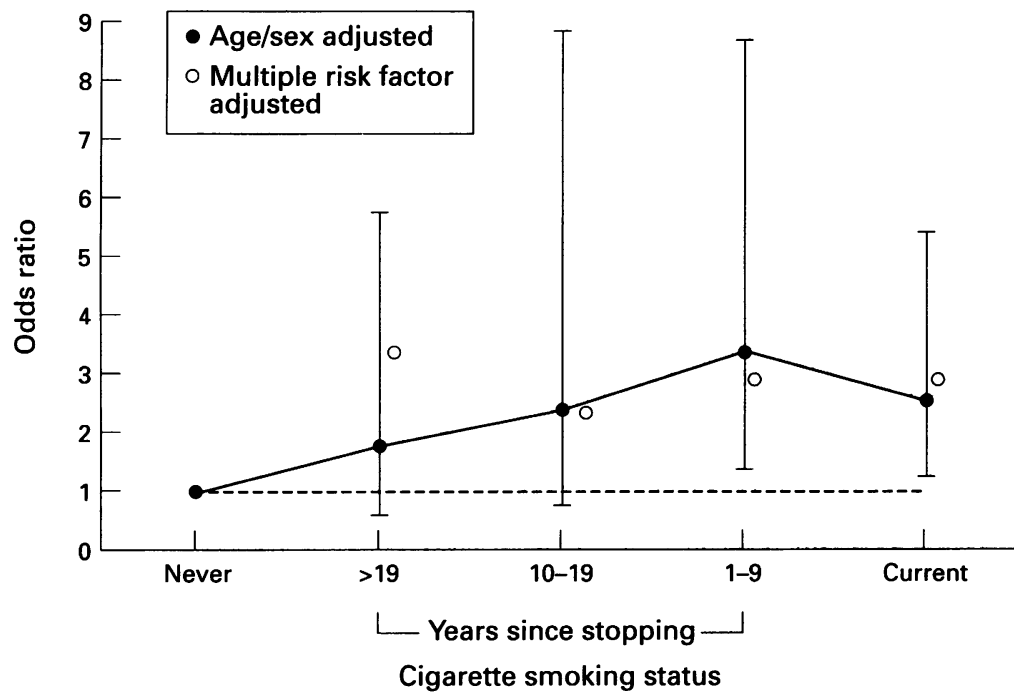

Figure 1 Relation between cigarette smoking and stroke (covariables included in the logistic models were; age group, sex, race [black/non-black], vigorous exercise aged 15-25 and dietary saturated fat [four levels]). Bars indicate $95 \%$ confidence intervals.

Table 2 Relation between cigarette smoking and stroke by sex

\begin{tabular}{llrr}
\hline & \multicolumn{3}{l}{ Cigarette smoking status (OR $(95 \%$ CI)) } \\
\cline { 2 - 4 } & Never & \multicolumn{1}{c}{ Former } & \multicolumn{1}{c}{ Current } \\
\hline Males: & 1.0 & $1.99(0.61,6.80)$ & $2.78(0.86,9.74)$ \\
$\quad$ Age adjusted & 1.0 & $2.87(0.91,9.10)$ & $3.23(0.99,10.5)$ \\
Multiple risk factor adjusted* & $6 / 17$ & $36 / 48$ & $31 / 33$ \\
$\quad$ Cases/controls & & & \\
Females: & 1.0 & $3.50(1.30,10.4)$ & $2.44(0.98,6.61)$ \\
$\quad$ Age adjusted & 1.0 & $3.95(1.51,10.3)$ & $3.38(1.32,8.67)$ \\
Multiple risk factor adjusted* & $16 / 52$ & $18 / 21$ & $18 / 27$ \\
Cases/controls & &
\end{tabular}

* Multiple risk factor adjustment included age group (4 age groups), race (black/non-black), vigorous exercise aged 15 to 25 (yes/no) and dietary saturated fat (4 levels) as covariables. race (black/non-black) tended to increase the size of the risk of both current and former smokers (fig 1, table 1). Smokers tend to be thinner and less hypertensive than never smokers but these observations may reflect an effect of smoking itself. Including markers of body fat and hypertension history in the adjusted analysis, therefore, would tend to falsely elevate the apparent risks of smoking. Separate univariate adjustment by the following had no appreciable effect on the relation between cigarette smoking and stroke: family history of stroke, social class, alcohol consumption, diabetes history, and cardiac ischaemia history. The hazards of smoking were seen in both sexes (table 2) and in all age groups (results not presented). When the data were analysed excluding surrogate responders, leaving 95 cases and 196 controls, there was no major change in the age/sex adjusted odds ratios; current -2.84 , ex 1-9 years -4.00 , ex $10-19$ years -3.88 , ex $>19$ years, 1.52 . When proven cerebral infarction was examined separately using the same methods of analysis as for overall stroke the results were similar to those for all stroke (results not presented).

A possible explanation for the high risk in those recently stopping smoking was that they had given up because of diagnosed vascular disease other than stroke. $18(67 \%)$ of the 27 stroke patients who had stopped smoking within the previous 10 years had a history of either; ischaemic heart disease, peripheral vascular disease, transient ischaemic attack, hypertension, or diabetes mellitus. This compared with $66(33 \%)$ of the 198 controls.

\section{LIFESTYLE COMBINATIONS AND POPULATION} ATTRIBUTABLE RISK FRACTION

Because lifestyle related risk factors such as smoking, lack of exercise, and excessive body fat are closely interrelated it was felt multiplying "independent" effects would be more likely to have mathematical appeal than to reflect true biological effects. Body fat, for example, is influenced by both physical exercise and cigarette smoking. For this reason lifestyle related risk factors in combination were assessed in order to examine their possible overall preventive potential for stroke. As cigarette smoking remains the best established lifestyle related risk factor it was used to form the basis of other combinations. If both former (prevalence in controls $=35 \%$ ) and current (prevalence in controls $=30 \%$ ) smoking were used to estimate the population attributable risk fraction, cigarettes appeared to be responsible for approximately half of all strokes in the population studied (table 3). The risk fraction con- 
Table 3 Relative risk and population attributable risk fraction for stroke of combinations of cigarette smoking history, exercise history, and history of obesity

\begin{tabular}{|c|c|c|c|c|}
\hline Variable & & $O R^{*}(95 \% C I)$ & Cases/controls & $\begin{array}{l}\text { Population attributable } \\
\text { risk fraction ( } 95 \% \text { CI) }\end{array}$ \\
\hline \multirow[t]{2}{*}{ Cigarette smoking } & Never & 1.0 & $22 / 69$ & \\
\hline & & $2.6(1.4,5.2)$ & $103 / 129$ & $0.49(0.22,0.67)$ \\
\hline Maximum reported BMI $†$ & $\begin{array}{l}\text { Quartiles } 1-3 \\
\text { Upper quartile }\end{array}$ & $1.7(1.0,2.8)$ & $46 / 50$ & $0.16(0.02,0.28)$ \\
\hline \multirow[t]{2}{*}{ Vigorous exercise aged 15- 25} & Yes & & $55 / 130$ & \\
\hline & No & $3.0(1.8,5.5)$ & $70 / 68$ & $0.33(0.16,0.46)$ \\
\hline \multirow{2}{*}{$\begin{array}{l}\text { Cigarette smoking and/or lack of vigorous } \\
\text { exercise aged 15-25 }\end{array}$} & No & 1.0 & $8 / 33$ & \\
\hline & Yes & $2.7(1.1,7.0)$ & $117 / 165$ & $0.62(0.20,0.82)$ \\
\hline \multirow{2}{*}{$\begin{array}{l}\text { Cigarette smoking and/or upper quartile } \\
\text { maximum reported BMI } \dagger\end{array}$} & No & & $8 / 45$ & \\
\hline & Yes & $4.3(1.9,12)$ & $116 / 151$ & $0.72(0.43,0.86)$ \\
\hline \multirow{2}{*}{$\begin{array}{l}\text { Cigarette smoking and/or lack of vigorous } \\
\text { exercise aged } 15 \text { to } 25 \text { and/or upper quartile } \\
\text { maximum reported BMI }\end{array}$} & No & 1.0 & $3 / 23$ & \\
\hline & Yes & $5.3(1.4,23)$ & $121 / 173$ & $0.79(0.32,0.94)$ \\
\hline
\end{tabular}

BMI = body mass index

* Odds ratio adjusted for age and sex.

† Data restricted to 124 cases and 196 controls with recall of weight. Upper quartile; males $->30.5 \mathrm{~kg} / \mathrm{m}^{2}, \mathrm{females}->31.3 \mathrm{~kg} /$ $\mathrm{m}^{2}$.

tributions of former (0.26) and current smoking (0.24) were roughly equal.

Data from this study have been published previously indicating physical activity at all stages of adult life offers protection against stroke. ${ }^{7}$ Cohort studies examining activity levels at the time of assessment are consistent with these observations. ${ }^{6}$ History of exercise aged 15-25 years was added to the analysed combination to represent physical activity both because it reflected lifelong exercise habits and because it was less likely to be influenced by illnesses in later life. ${ }^{7}$ This combination of cigarette smoking and/or lack of exercise increased the attributable risk fraction to $62 \%$ (table 3). The age/sex and multiple risk factor adjusted (for age group, sex, race, maximum reported body mass index and dietary saturated fat) odds ratios for this combination were similar -2.73 and 2.83 respectively.

Overweight and obesity are now more widely accepted as stroke risk factors which reflect dietary and exercise habits. ${ }^{58}$ In this study the most appropriate marker of obesity and overweight appeared to be recall of lifelong maximum body mass index. A combination, therefore, of cigarette smoking and/or maximum reported body mass index in the upper quartile was examined. This combination accounted for $72 \%$ of all strokes (table 3 ). The age/sex and multiple risk factor adjusted (for age group, sex, race, exercise aged 15-25, and dietary saturated fat) odds ratios for this combination were, again, similar -4.31 and 4.49 respectively. If upper quartile of subscapular skinfold thickness was added to cigarette smoking in place of maximum reported body mass index similar figures were reached: odds ratio for cigarette smoking and/or upper quartile of subscapular skinfold thickness compared with thinner never smokers - $3.45(1.55,7.43)$. It is of note that only one stroke patient reported never smoking and remaining in the lowest maximum reported quartile of body mass index $\left(<25 \mathrm{~kg} / \mathrm{m}^{2}\right)$.

Combining cigarette smoking, a history of obesity and lack of exercise increased the population attributable risk fraction towards $80 \%$ (table 3). As before the age/sex and multiple risk factor adjusted (for age group, sex, race, and dietary saturated fat) odds ratios for this combination were similar -5.29 and 5.94 respectively. It is, finally, worth noting that no stroke patient reported a combination of never smoking, never being overweight $\left(>25 \mathrm{~kg} / \mathrm{m}^{2}\right)$ and taking regular vigorous exercise aged 1525.

\section{Discussion}

The results indicate the substantial potential for stroke prevention which could be achieved through easily identifiable measures related to lifestyle.

\section{CIGARETTE SMOKING}

The results of this study confirm that current cigarette smokers are two to three times more likely to have a stroke than those who have never smoked. The study, also, suggests that the risks of cigarette smoking for stroke last one, two, or possibly more decades after subjects have given up smoking. The calculated population attributable risk for a history of cigarette smoking indicates that around half of all strokes in the age group and population studied could have been prevented if people had never started smoking.

The persistent nature of the risk of cigarette smoking for stroke in this study is consistent with other studies of both stroke in those under 75 years of age and cerebral infarction. ${ }^{12}$ Another British case-control study found a similar slow decline in risk but a smaller overall risk amongst female former smokers. ${ }^{15}$ The findings are also consistent with the results of the British regional heart study for myocardial infarction - another disease related to atherosclerosis. ${ }^{16}$ For stroke the latter study revealed only a minimal excess risk in former smokers, although confidence intervals would overlap with those reported here. ${ }^{3}$ The weaker impact of former smoking seen in the nurses cohort may be partly explained by the younger age/fewer pack years that would have been prevalent amongst their former smokers. ${ }^{4}$ 
The risks of current and past smoking are more clearly seen in this study than in some others. There are several possible reasons for this that relate to difficulties that can arise with cohort and hospital based case-control studies. Accurate case definition is a potential problem in large cohort studies as clinical information from possibly less reliable sources has to be used, for example; death certificates, patient recall or a review of clinical records. This is a particular problem as age increases - most cohort studies have included a substantial proportion of elderly stroke patients. Misclassification in these studies would have tended to attenuate the real risks. Another problem raised by cohort studies is the incomplete nature of the exposure history; current smokers at screening may be formersmokers by the time the stroke occurs. Hospital based case-control studies may also underestimate the true risks of cigarette smoking due to selection biases operating on the control subjects. This is because it is quite plausible that those with a history of cigarette smoking are more likely to be under some form of hospital care and thus available to act as a control. This study offered a detailed assessment of all cases in a defined population by a physician, an up to date history of exposure to cigarette smoking and minimal selection biases because of the almost complete response rate and the community based nature of the study. Examination of smoking history recorded in general practice/hospital notes prior to assessment in the study provided little evidence of recall bias. Pipe and cigar smoking will not have exerted any appreciable influence on the results as these habits were virtually unknown in this population (reported by one case and one control).

\section{COMBINATIONS OF LIFESTYLE RELATED RISK FACTORS}

Taken together, the combination of cigarette smoking, excessive body fat, and lack of regular vigorous exercise may have accounted for between 70 and $80 \%$ of stroke cases in the population studied. The size of the preventive potential indicated by these results has not been previously described. This estimate can only be a guide as the $95 \%$ CI are wide and the excluded stroke patients have not been incorporated into the figure.

Potential sources of error in a study such as this may have come from misclassification of the exposures and confounding, as well as the limited size of the study. There is good evidence on the accuracy of cigarette smoking classification in this study and data previously published are reassuring as regards misclassification in relation to lifelong body fat. ${ }^{8}$ It is more difficult to be confident about reports of previous exercise but the contribution of the latter to the overall combined population attributable risk fraction is small. Any unbiased misclassification in the assessment of exercise (potentially considerable) will have led to an underestimate of its impact. If a biased tendency existed for stroke patients to reply in the negative about past exercise the attributable risk fraction could have been falsely inflated.

It seems unlikely that the results were due to confounding by any well established risk factors. If unadjusted, age and sex adjusted or multiple risk factor adjusted odds ratios were calculated for the three combined lifestyle related factors the odds ratio remained the same. Although hypertension, diabetes, and cardiac disease are known to be associated with both obesity and stroke, it is difficult to make the case that these risk factors should be regarded as potentially important confounders. Hypertension, diabetes, and cardiac disease are likely to lie in the causal pathway of stroke as clinical syndromes which result from both genetic and environmental influences. This study was directed towards the latter potentially avoidable risks which could be more easily identifiable by the population at large. Hypertension, diabetes, and heart disease all require identification by a clinician - cigarette smoking, overweight, and inactivity do not. Data from the prospective studies collaboration do indicate that diastolic blood pressures greater than $70 \mathrm{mmHg}$, if considered causal (data were unadjusted), account for around $60 \%$ of all stroke cases. ${ }^{17} \mathrm{~A}$ recent report from the Framingham cohort, however, notes that around $70 \%$ of hypertension is directly attributed to obesity. ${ }^{18}$ These inter-relations mean that high population attributable risk fractions for both hypertension and the lifestyle related factors addressed in this study are likely to be compatible. For these reasons results adjusted for hypertension, diabetes, and cardiac ischaemia were not computed. Rothman argues it is inappropriate to adjust for variables in the causal pathway except to explore the mechanisms involved. ${ }^{19}$ The size of any contribution of specific dietary intakes (eg salt), interuterine nutrition, or genetic predisposition to the population attributable risk fraction for stroke remains unclear. A degree of confounding by these variables is possible but unlikely to be substantial. Problems with assessment of these variables and limitations on the interpretation of blood pressure measurement in patients presenting with an acute stroke mean some of these inter-relationships are not easily explored in studies of a case-control design.

The results of this study, particularly if taken together with the results of the Whitehall study, suggest that there is an interaction between the effects of cigarette smoking and body fat. ${ }^{5}$ Dangers, therefore, would exist in merely adding or multiplying these two risks together to obtain a combined effect. A more reliable (although statistically low powered) approach was, therefore, adopted by examination of the risk factors in combination. An approach to combinations using regression models would, perhaps, tend to underestimate the preventive scope of these two potentially avoidable hazards.

The data of American college alumni, using combinations of cigarette smoking and overweight are consistent with the findings of this study - smoking/overweight students had a stroke morbidity ratio of 2.6 compared with 
other colleagues. ${ }^{20}$ The stroke patients in the alumni study, however, were self reported and exposures had only been measured once, around the age of 20 .

The observation that a history of inactivity does not appear to contribute much over and above a history of cigarette smoking is worth comment. There was a trend for the benefit of exercise to be greater amongst cigarette smokers ${ }^{7}$ but the small number of never smoking stroke patients in this study means that it is difficult to draw conclusions until the potential benefits of exercise in a larger never smoking population have been assessed.

The finding that a substantial proportion of strokes in the age group studied could probably be prevented by avoidance of cigarettes, excessive body fat, and inactivity has implications for health promotion. It conveys a positive message to younger people about the potential to avoid premature cerebrovascular disease. It is also consistent with recent findings about the limitations of advising and encouraging people to stop smoking and lose weight in later adult life. ${ }^{21}$ The results support the current preventive strategy addressing these risks but add weight to the case for concentrating efforts at those in younger age groups. Working towards creating an environment in which it is easy to adopt these health promoting patterns of living would seem to offer large gains for stroke prevention.

The results highlight particular concern for long term cardiovascular health from the increasing prevalence of obesity in western populations and the associated decrease in activity levels. The fall in stroke mortality attributed to declining smoking in men, better management of hypertension, anticoagulation for atrial fibrillation, carotid endarterectomy and aspirin use could be reversed. The situation is even more alarming in developing countries where smoking is also increasingly prevalent.

I thank the patients and staff of the 11 participating general practices (see below) for their cooperation, Professor D G Beevers and Dr I J Perry for their advice, and Dr G Sagar for computed tomography. The project was funded by the Wellcome Trust. The participating practices: Bearwood Road Surgery; Srust. The participating practices: Bearwood Road Surgery; Smethwick, Birchfield Medical Centre, Cape Hill Medical Centre, Church Lane Surgery; Handsworth, Hamstead Road Surgery; Handsworth /Great Barr, Lee Bank Health Centre, Newtown Health Centre, Plough and Harrow Medical Centre;
Ladywood, Sherwood House Surgery; Bearweed, Tower Hill Ladywood, Sherwood House Surgery; Bearweed,
Health Centre, Victoria Health Centre; Smethwick.

1 Shinton R, Beevers G. Meta-analysis of the relation between cigarette smoking and stroke. BMf 1989;298:789-94.

2 Donnan GA, Adena MA, O'Malley HM, McNeil JJ, Doyle AE, Neill GC. Smoking as a risk factor for cerebral AE, Neill GC. Smoking as a ris

3 Shaper AG, Phillips AN, Pocock SJ, Walker M, Macfarlane PW. Risk factors for stroke in middle-aged British men. BMF 1991;302:1111-5.

4 Kawachi I, Colditz GA, Stampfer MJ, Smoking cessation and decreased risk of stroke in women. $\mathcal{F} A M A$ 1993;269: $232-6$.

5 Shinton $R$, Shipley $M$, Rose G. Overweight and stroke in the Whitehall study. $f$ Epidemiol Community Health 1991; 45:138-42.

6 Wannamethee G, Shaper AG. Physical activity and stroke in middle aged British men. $B M \mathcal{F}$ 1992;304:597-601

7 Shinton R, Sagar G. Lifelong exercise and stroke. $B M \mathcal{F}$ 1993;307:231-4

8 Shinton R, Sagar G, Beevers G. Body fat and stroke: Unmasking the hazards of overweight and obesity. $\mathcal{F}$ Epidemiol Community Health 1995;49:259-64.

9 Shinton R, Sagar G, Beevers G. The relation of alcohol consumption to cardiovascular risk factors and stroke. The west Birmingham stroke project. $\mathcal{f}$ Neurol Neurosurg The west Birmingham stro
Psychiatry 1993;56:458-62.

10 World Health Organisation. Cerebrovascular diseases - prevention, treatment and rehabilitation. Technical report series no. 469. WHO: Geneva, 1971

11 Weiner JS, Lourie JA. Human Biology: a guide to field methods. IBP Handbook No.9. Oxford: Blackwell Scientific Publications, 1969:27-52.

12 EGRET. Reference manual. Seattle: Statistics and epidemiology research corporation, 1990

13 Breslow NE, Day NE. Statistical methods in cancer research. Volume I-The analysis of Case-control studies. Lyon: International agency for research on cancer, 1980:122-159.

14 Schlesselman JJ. Case-Control Studies. Design, Conduct and Analysis, New York 1982:220-222.

15 Thompson SG, Greenberg G, Meade TW. Risk factors for stroke and myocardial infarction in women in the United Kingdom as assessed in general

16 Cook DG, Shaper AG, Pocock SJ, Kussick SJ. Giving up smoking and the risk of heart attacks. A report from the British Regional Heart Study. Lancet 1986; ii:1376-80.

17 Prospective Studies Collaboration. Cholesterol, diastolic Prospective Studies Collaboration. Cholesterol, diastolic
blood pressure, and stroke: 13000 strokes in 450000 people in 45 prospective cohorts. Lancet 1995;346:164753.

18 Kannel WB. Blood pressure as a cardiovascular risk factor. Prevention and treatment. $\mathcal{F A M A}$ 1996;275:1571-1576.

19 Rothman KJ. Modern Epidemiology. Little Brown and Copmany, Boston 1986:89-94

20 Paffenbarger RS, Wing AL. Chronic disease in former college students: early precursors of non-fatal stroke. $\mathrm{Am} \mathcal{F}$ Epidemiol 1971;94:524-30.

21 Multiple Risk Factor Intervention Trial Group. Multiple risk factor intervention trial: risk factor changes and mortality

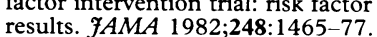

